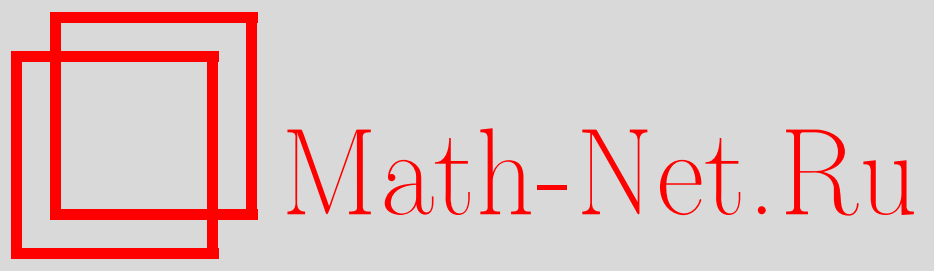

А. А. Славнов, О возможности описания неабелевых массивных калибровочных полей в рамках перенормируемой теории, ТМФ, 2017, том 193, номер 3, 484-492

DOI: https://doi.org/10.4213/tmf9429

Использование Общероссийского математического портала Math-Net.Ru подразумевает, что вы прочитали и согласны с пользовательским соглашением http://www.mathnet.ru/rus/agreement

Параметры загрузки:

IP: 54.224 .60 .19

26 апреля 2023 г., 03:08:40

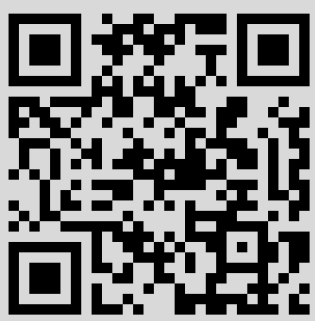




\title{
О ВОЗМОЖНОСТИ ОПИСАНИЯ НЕАБЕЛЕВЫХ МАССИВНЫХ КАЛИБРОВОЧНЫХ ПОЛЕЙ В РАМКАХ ПЕРЕНОРМИРУЕМОЙ ТЕОРИИ
}

\begin{abstract}
Предложена перенормируемая теория массивных неабелевых калибровочных полей, которая не требует существования наблюдаемых скалярных полей.
\end{abstract}

Ключевые слова: массивные неабелевы калибровочные поля.

DOI: https://doi.org/10.4213/tmf9429

\section{1. ВВЕДЕНИЕ}

Хотя открытие в ЦЕРН частицы, которая, вероятно, может играть роль скалярного мезона, предсказанного в работах [1], [2], и которая лежит в основе электрослабого сектора Стандартной модели [3]-[5], частично решило вопрос о справедливости Стандартной модели, построение перенормируемой модели, включающей только массивные калибровочные поля, остается весьма актуальным.

Недавно в наших работах [6]-[?] была предложена новая формулировка теории Янга-Миллса, которая свободна от проблемы грибовского произвола [13] и, следовательно, может быть использована как в рамках теории возмущений, так и вне нее. Эта формулировка позволяет преодолеть теорему об отсутствии солитонных решений в теории Янга-Миллса; были построены в явном виде классические решения, соответствующие солитонам.

В настоящей работе мы показываем, что небольшая модификация данного формализма позволяет описывать в рамках перенормируемой теории поля массивные калибровочные поля без введения наблюдаемых скалярных полей.

Работа построена следующим образом. В разделе 2 представлен лагранжиан, описывающий новую теорию, и обсуждаются проблемы, связанные с ее перенормируемостью. В разделе 3 доказана унитарность рассматриваемой теории в физическом пространстве. В разделе 4 обсуждаются некоторые проблемы, связанные с применением данной теории к описанию моделей электрослабого взаимодействия.

Исследование выполнено за счет гранта Российского научного фонда (проект № 14-50-00005).

* Математический институт им. В. А. Стеклова Российской Академии наук, Москва, Россия. E-mail: slavnov@mi.ras.ru 


\section{2. ФОРМУЛИРОВКА МОДЕЛИ}

Мы начинаем с лагранжиана, описывающего, как показано в работе [6], безмассовое поле Янга-Миллса:

$$
\begin{aligned}
L= & -\frac{1}{4} F_{\mu \nu}^{a} F_{\mu \nu}^{a}+\left(D_{\mu} \varphi_{+}\right)^{*}\left(D_{\mu} \varphi_{-}\right)+\left(D_{\mu} \varphi_{-}\right)^{*}\left(D_{\mu} \varphi_{+}\right)+ \\
& +\left(D_{\mu} b\right)^{*}\left(D_{\mu} e\right)+\left(D_{\mu} e\right)^{*}\left(D_{\mu} b\right),
\end{aligned}
$$

где $\varphi_{ \pm}$- это бозе-поля, a $b, e$ - антикоммутирующие элементы алгебры Грассмана. Для краткости мы будем рассматривать калибровочную группу $S U(2)$, при этом поля $\varphi_{ \pm}, b$, е являются комплексными дублетами, реализующими фундаментальное представление этой группы,

$$
\varphi_{ \pm}(x) \rightarrow \varphi_{ \pm}^{\omega}=\Gamma[\omega(x)] \varphi_{ \pm}(x), \quad \Gamma[\omega(x)]=\frac{i g}{2} \omega^{a} \tau^{a} .
$$

Аналогичные формулы справедливы для полей $b, e$.

В секторе, в котором духовые поля отсутствуют, в асимптотических состояниях континуальный интеграл по духовым полям $\varphi_{ \pm}, b, e$ можно вычислить явно и убедиться, что остающийся континуальный интеграл совпадает с соответствующим интегралом в стандартной теории Янга-Миллса. Это простое доказательство, однако, не проходит в случае, когда асимптотические состояния включают духовые частицы. Тем не менее ниже будет показано, что теория может быть унитарна в пространстве, которое содержит в асимптотических состояниях только наблюдаемые частицы, при условии, что имеется некоторая дополнительная симметрия, отсутствующая в стандартной формулировке.

Наша теория включает помимо неабелевых калибровочных полей также комплексные скалярные дублеты $\varphi_{ \pm}, b, e$, которые можно параметризовать (анти)эрмитовыми компонентами:

$$
\Phi=\left(\frac{i \Phi_{1}+\Phi_{2}}{\sqrt{2}}, \frac{\Phi_{4}-i \Phi_{3}}{\sqrt{2}}\right), \quad \Phi^{*}=\left(\frac{-i \Phi_{1}+\Phi_{2}}{\sqrt{2}}, \frac{\Phi_{4}+i \Phi_{3}}{\sqrt{2}}\right) .
$$

Компоненты поля $b$ считаются антиэрмитовыми.

В отличие от предыдущих работ, здесь мы будем считать, что поля $\varphi_{ \pm}$имеют нетривиальные асимптотики на бесконечности:

$$
\varphi_{ \pm} \rightarrow \frac{\hat{\alpha} m}{g}, \quad \varphi_{ \pm}^{*} \rightarrow \frac{\hat{\alpha} m}{g} .
$$

Введем переменные $\tilde{\varphi}_{ \pm}$, имеющие нулевую асимптотику на бесконечности, с помощью формулы

$$
\varphi_{ \pm}=\tilde{\varphi}_{ \pm}+\frac{\hat{\alpha} m}{g}, \quad \varphi_{ \pm}^{*}=\tilde{\varphi}_{ \pm}^{*}+\frac{\hat{\alpha} m}{g} .
$$

Мы считаем, что поля $b$, е убывают на бесконечности достаточно быстро. В терминах полей $\tilde{\varphi}_{ \pm}$лагранжиан (1) имеет вид

$$
\begin{aligned}
L= & -\frac{1}{4} F_{\mu \nu}^{a} F_{\mu \nu}^{a}+\left(D_{\mu} \tilde{\varphi}_{+}\right)^{*}\left(D_{\mu} \tilde{\varphi}_{-}\right)+\left(D_{\mu} \tilde{\varphi}_{-}\right)^{*}\left(D_{\mu} \tilde{\varphi}_{+}\right)+\frac{m^{2}}{2} A_{\mu}^{2}+ \\
& +\left\{\frac{m}{g}\left(D_{\mu} \hat{\alpha}_{+}\right)^{*}\left(D_{\mu} \tilde{\varphi}_{-}\right)+\frac{m}{g}\left(D_{\mu} \hat{\alpha}_{-}\right)^{*}\left(D_{\mu} \tilde{\varphi}_{+}\right)+\text {э.c. }\right\}+ \\
& +\left(D_{\mu} e\right)^{*}\left(D_{\mu} b\right)+\left(D_{\mu} b\right)^{*}\left(D_{\mu} e\right) .
\end{aligned}
$$


В этом выражении $\hat{\alpha}_{ \pm}=(0, \alpha), \alpha^{2}=1 / 2$. Преобразование (5) представляет собой замену переменных, следовательно, "сдвинутый" лагранжиан инвариантен относительно “сдвинутых" калибровочных преобразований. В терминах (анти)эрмитовых компонент эти преобразования имеют вид

$$
\begin{aligned}
\delta A_{\mu}^{a}=\partial_{\mu} \eta^{a}+g \epsilon^{a b c} A_{\mu}^{b} \eta^{c}, & \\
\delta \tilde{\varphi}_{ \pm}^{a}=\frac{\alpha_{ \pm} m}{\sqrt{2}} \eta^{a}+\frac{g}{2} \epsilon^{a b c} \tilde{\varphi}_{ \pm}^{b} \eta^{c}+\frac{g}{2} \tilde{\varphi}_{ \pm}^{0} \eta^{a}, & \delta \tilde{\varphi}_{ \pm}^{0}=-\frac{g}{2} \tilde{\varphi}_{ \pm}^{a} \eta^{a} \\
\delta b^{a}=\frac{g}{2} \epsilon^{a b c} b^{b} \eta^{c}+\frac{g}{2} b^{0} \eta^{a}, & \delta b^{0}=-\frac{g}{2} b^{a} \eta^{a} \\
\delta e^{a}=\frac{g}{2} \epsilon^{a b c} e^{b} \eta^{c}+\frac{g}{2} e^{0} \eta^{a}, & \delta e^{0}=-\frac{g}{2} e^{a} \eta^{a} .
\end{aligned}
$$

Асимптотический лагранжиан инвариантен относительно более простых преобразований

$$
\delta A_{\mu}^{a}=\partial_{\mu} \eta^{a}, \quad \delta \tilde{\varphi}_{ \pm}^{a}=\frac{\alpha_{ \pm}}{\sqrt{2}} m \eta^{a}
$$

остальные асимптотические поля не меняются. Видно, что в модифицированной теории имеются три поля, сдвигающиеся при калибровочных преобразованиях на произвольную функцию, т. е. вместо одного калибровочного поля мы имеем три калибровочных поля. Чтобы проквантовать лагранжиан (6), необходимо выбрать условие калибровки. В настоящей работе мы главным образом интересуемся теорией возмущений, в которой неоднозначность квантования отсутствует, поэтому мы выберем калибровку Лоренца $\partial_{\mu} A_{\mu}^{a}=0$ и введем соответствующие духовые поля. В этой калибровке квадратичный член, который присутствует в лагранжиане (6) и имеет форму $A_{\mu}^{a}(x) \tau_{\gamma 2}^{a} \partial_{\mu} \varphi_{\gamma}(x)$, не дает вклада, так как уравнение для поля $A$ выглядит следующим образом:

$$
\partial_{\mu}\left(\partial_{\mu} A_{\nu}^{a}-\partial_{\nu} A_{\mu}^{a}\right)+m^{2} A_{\nu}^{a}=0, \quad \partial_{\nu} A_{\nu}^{a}=0 .
$$

Теперь эффективное действие имеет вид

$$
S_{\mathrm{eff}}=\int d x\left[L+\lambda^{a} \partial_{\mu} A_{\mu}^{a}+i \partial_{\mu} \bar{c} D_{\mu} c\right],
$$

где лагранжиан $L$ определен формулой (6).

Очевидно, действие (10) соответствует перенормируемой теории, поскольку все пропагаторы убывают на бесконечности, как $k^{-2}$, а взаимодействие включает только тройной член с одной производной и четверной член без производных.

\section{3. УНИТАРНОСТЬ В ФИЗИЧЕСКОМ ПРОСТРАНСТВЕ}

Задача рассеяния в квантовой теории формулируется следующим образом. При $t \rightarrow \pm \infty$ поля описываются свободными уравнениями движения и в процессе рассеяния измеряется вероятность перехода из одного асимптотического состояния в другое. Разумеется, возможна некоторая перестройка физического асимптотического состояния. Асимптотическое состояние определяется граничными условиями, наложенными на матрицу рассеяния. Унитарность будет доказана, если мы установим эквивалентность нашей модели в физическом пространстве стандартной теории, в которой матрица рассеяния заведомо унитарна. Для исследования вопроса об унитарности достаточно рассмотреть асимптотическую теорию. 
Асимптотическое эффективное действие (10) инвариантно относительно преобразований суперсимметрии

$$
\delta \tilde{\varphi}_{-}=-i b \epsilon, \quad \delta e=\tilde{\varphi}_{+}+\text {const }, \quad \delta b=\delta \tilde{\varphi}_{+}=0 .
$$

Эти преобразования являются асимптотическим пределом преобразований, которые оставляют эффективное действие (10) неизменным. Они получаются с помощью замены переменных (5) в асимптотическом эффективном действии (10) и, очевидно, меняют асимптотику поля $e$. Поскольку эти преобразования меняют асимптотическое поведение поля $e^{0}$, создается впечатление, что они изменяют пространство, в котором определена теория, и, следовательно, недопустимы. В данном разделе мы показываем, что симметрия асимптотического действия обеспечивает унитарность матрицы рассеяния в физическом подпространстве, в результате рассматриваемая нами модель имеет матрицу рассеяния, унитарную в подпространстве, совпадающем с пространством наблюдаемых в массивной теории Янга-Миллса. Такая модель может использоваться для описания массивной неабелевой калибровочной теории.

Действие (6) инвариантно относительно калибровочных преобразований. Следовательно, после фиксации калибровки оно инвариантно относительно преобразований БРСТ, которые можно получить из преобразований (7) заменой $\eta^{a}$ на $i c^{a} \epsilon$, где $\epsilon$ - постоянный антикоммутирующий параметр. Эффективное действие (10) БРСТинвариантно, если поля $\lambda, \bar{c}, c$ обладают следующими трансформационными свойствами:

$$
\delta \lambda^{a}=0, \quad \delta \bar{c}^{a}=\lambda^{a} \epsilon, \quad \delta c^{a}=\frac{g}{2} \epsilon^{a b d} c^{b} c^{d} \epsilon .
$$

Естественный выбор физического подпространства дают состояния, удовлетворяющие условиям

$$
Q_{\mathrm{B}}|\psi\rangle_{\mathrm{ph}}=0, \quad Q_{\mathrm{S}}|\psi\rangle_{\mathrm{ph}}=0 ;
$$

здесь $Q_{\text {в }}$ - это БРСТ-инвариантный заряд, сохраняющийся по теореме Нётер вследствие инвариантности эффективного действия относительно преобразований БРСТ. Оператор $Q_{\mathrm{S}}$ представляет аналогичный заряд, сохраняющийся благодаря инвариантности эффективного действия относительно преобразований суперсимметрии. Но преобразования суперсимметрии плохо определены. Согласно приведенным выше аргументам достаточно рассматривать вместо уравнений (13) соответствующие уравнения для асимптотических состояний, т. е. состояний, которые удовлетворяют свободным уравнениям, но дают наблюдаемые значения масс и зарядов.

Асимптотические условия наложены на физические поля, духовые поля аннигилируются нётеровским БРСТ-зарядом $Q_{\mathrm{B}}^{\mathrm{as}}$ и суперзарядом $Q_{\mathrm{S}}^{\mathrm{as}}$ :

$$
Q_{\mathrm{B}}^{\mathrm{as}}|\psi\rangle_{\mathrm{ph}}^{\mathrm{as}}=0, \quad Q_{\mathrm{s}}^{\mathrm{as}}|\psi\rangle_{\mathrm{ph}}^{\mathrm{as}}=0, \quad\left[Q_{\mathrm{B}}^{\mathrm{as}}, Q_{\mathrm{S}}^{\mathrm{as}}\right]_{+}=0 .
$$

Здесь, однако, имеется проблема. Дело в том, что теорема Нётер гарантирует существование сохраняющегося тока $\partial_{\mu} J_{\mu}=0$, если действие инвариантно относительно некоторого преобразования полей и их производных, но не гарантирует существования соответствующего сохраняющегося заряда. Проблема корректного определения инвариантности теории была рассмотрена в книге [14], здесь мы ограничимся замечанием, что в данной модели существует хорошо определенный ток, который асимптотически сохраняется. 
Можно заменить $Q_{\mathrm{S}}^{\text {as }}$ хорошо определенным асимптотически сохраняющимся оператором

$$
\widetilde{Q}_{\mathrm{S}}^{\mathrm{as}}=\int d^{3} x\left(\partial_{0} \tilde{\varphi}_{+}^{\mathrm{as}, \alpha} b^{\mathrm{as}, \alpha}-\tilde{\varphi}_{+}^{\mathrm{as}, \alpha} \partial_{0} b^{\mathrm{as}, \alpha}+\text { э.c. }\right), \quad \alpha=0,1,2,3 .
$$

Мы напоминаем читателю, что согласно принятому определению поле $b$ является антиэрмитовым. Этот оператор существует, так как он квадратичен по полям. Асимптотическое сохранение оператора $\widetilde{Q}_{\mathrm{S}}^{\mathrm{as}}$ можно легко проверить непосредственно, поскольку поля $b^{\text {as }}, \tilde{\varphi}_{+}^{\text {as }}$ удовлетворяют уравнениям

$$
\partial^{2} b^{\text {as }}=\partial^{2} b^{\text {as }, *}=\partial^{2} \tilde{\varphi}_{+}^{\text {as }}=\partial^{2} \tilde{\varphi}_{-}^{\text {as }}=0 .
$$

Оператор (15), очевидно, является нильпотентным.

Как следует из гипотезы об адиабатическом выключении взаимодействия,

$$
\lim _{t \rightarrow \infty} U^{-1}(t) H U(t)=H^{\text {as }}
$$

где оператор $U(t)$ задан как

$$
U(t)=\exp \left\{i H^{\mathrm{as}} t\right\}
$$

Введем оператор $\widetilde{Q}_{\mathrm{S}}(t)$, который асимптотически совпадает с $\widetilde{Q}_{\mathrm{s}}^{\mathrm{as}}$ :

$$
\lim _{|t| \rightarrow \infty} U^{-1} Q_{\mathrm{S}}(t) U=Q_{\mathrm{S}}^{\mathrm{as}} .
$$

Следующее равенство вытекает из того, что $\left[Q_{\mathrm{S}}^{\mathrm{as}}, H_{\mathrm{as}}\right]_{-}=0$ :

$$
\lim _{t \rightarrow \infty}\left[\widetilde{Q}_{\mathrm{S}}, H\right]_{-}=0 \text {. }
$$

Оператор $\widetilde{Q}_{\mathrm{S}}$ коммутирует с $H$ при $t \rightarrow \infty$. Оператор $U(t)$ можно ввести не только в пределе $t \rightarrow \infty$, но и для конечных $t$. В общем случае оператор $Q_{\mathrm{S}}(t)$ нелокален, но он нам требуется лишь при $|t| \rightarrow \infty$. Унитарность матрицы рассеяния в пространстве векторов, аннигилируемых оператором $\widetilde{Q}_{\mathrm{S}}^{\mathrm{as}}$, вытекает из следующих формальных выкладок [15], [16]:

$$
\begin{aligned}
S \widetilde{Q}_{\mathrm{S}}^{\mathrm{as}} & =\exp \left\{i H_{\mathrm{as}} t^{\prime \prime}\right\} \exp \left\{-i H\left(t^{\prime \prime}-t^{\prime}\right)\right\} \exp \left\{-i H_{\mathrm{as}} t^{\prime}\right\} \widetilde{Q}_{\mathrm{S}}^{\mathrm{as}}= \\
& =\exp \left\{i H_{\mathrm{as}} t^{\prime \prime}\right\} \exp \left\{-i H\left(t^{\prime \prime}-t^{\prime}\right)\right\} \widetilde{Q}_{\mathrm{S}} \exp \left\{-i H_{\mathrm{as}} t^{\prime}\right\}= \\
& =\exp \left\{i H_{\mathrm{as}} t^{\prime \prime}\right\} \widetilde{Q}_{\mathrm{S}} \exp \left\{-i H\left(t^{\prime \prime}-t^{\prime}\right)\right\} \exp \left\{-i H_{\mathrm{as}} t^{\prime}\right\}=\widetilde{Q}_{\mathrm{S}}^{\text {as }} S .
\end{aligned}
$$

Разумеется, это доказательство не исключает возможности некоторой перестройки физических асимптотических состояний. В соотношениях (21) подразумевается предел $t^{\prime \prime} \rightarrow \infty, t^{\prime} \rightarrow-\infty$. Как мы видели, достаточным условием унитарности матрицы рассеяния является равенство (20).

Оператор $\widehat{K}^{\mathrm{s}}$ допускает аналогичное представление. Можно взять

$$
\widehat{K}^{\mathrm{s}}=\int d^{3} x\left(\partial_{0} \tilde{\varphi}_{-}^{\mathrm{as}, \alpha} e^{\mathrm{as}, \alpha}-\tilde{\varphi}_{-}^{\mathrm{as}, \alpha} \partial_{0} e^{\mathrm{as}, \alpha}+\text { э.c. }\right), \quad \alpha=0,1,2,3 .
$$


Здесь символами $\tilde{\varphi}_{ \pm}$, e обозначены операторы рождения и уничтожения соответствующих полей, введенные формулами

$$
\begin{aligned}
& \varphi_{ \pm}(\mathbf{x}, t)=\frac{1}{2 \pi^{3 / 2}} \int \frac{d k}{\sqrt{2 \omega}}\left[a_{ \pm}^{*}(\mathbf{k}) e^{-i \mathbf{k} \mathbf{x}} e^{i k_{0} t}+a_{ \pm}(\mathbf{k}) e^{i \mathbf{k x}} e^{-i k_{0} t}\right] \\
& \pi_{ \pm}(\mathbf{x}, t)=\frac{1}{2 \pi^{3 / 2}} \int \frac{d k i \sqrt{\omega}}{\sqrt{2}}\left[a_{ \pm}^{*}(\mathbf{k}) e^{-i \mathbf{k x}} e^{i k_{0} t}-a_{ \pm}(\mathbf{k}) e^{i \mathbf{k x}} e^{-i k_{0} t}\right]
\end{aligned}
$$

где $\omega=k_{0}=\sqrt{\mathbf{k}^{2}}$. Видно, что

$$
\left[Q_{\mathrm{s}}^{\mathrm{as}}, \widehat{K}^{\mathrm{s}}\right]_{+}=Q_{\mathrm{s}}^{\mathrm{as}} \widehat{K}^{\mathrm{s}}+\widehat{K}^{\mathrm{s}} Q_{\mathrm{s}}^{\mathrm{as}}
$$

Замечая, что любой физический вектор аннигилируется операторами $Q_{\mathrm{S}}^{\mathrm{as}}$ и $K^{\mathrm{s}}$, мы видим, что любой физический вектор имеет структуру

$$
|\psi\rangle_{\mathrm{ph}}=|\psi\rangle_{\widetilde{\mathrm{ph}}}+|N\rangle,
$$

где вектор $|\psi\rangle_{\widetilde{\mathrm{ph}}}$ содержит физические возбуждения массивной теории Янга-Миллса и поля, соответствующие множителю Лагранжа $\lambda$, а также духи Фаддеева-Попова $\bar{c}, c$. Вектор $|N\rangle$ обозначает произвольный вектор с нулевой нормой, содержащий $N$ духовых частиц.

Проиллюстрируем этот факт простым примером. Рассмотрим процесс, при котором две векторные частицы переходят в две векторные частицы в ведущем порядке теории возмущений. Рассмотрим те члены, квадратичные по $A_{\mu}^{a}$, которые меняются, когда пропагатор векторной частицы становится поперечным. Члены второго порядка содержатся в выражении

$$
\int d x \epsilon^{a b c} f_{\mu \nu}^{a} A_{\mu}^{b} A_{\nu}^{c} \int d y \epsilon^{d e f} f_{\lambda \rho}^{d} A_{\lambda}^{e} A_{\rho}^{f} .
$$

Вклад таких членов в матрицу рассеяния с точностью до множителя равен

$$
\int d x \epsilon_{a b c} A_{\mu}^{a} A_{\nu}^{b}\left(\partial_{\mu} A^{c}(x)_{\nu}-\partial_{\nu} A^{c}(x)_{\mu}\right) \int d y \epsilon_{d e f} A_{\rho}^{d} A_{\sigma}^{e}\left(\partial_{\rho} A^{f}(y)_{\sigma}-\partial_{\sigma} A^{f}(y)_{\rho}\right) .
$$

Мы хотим преобразовать это выражение к поперечной калибровке, в которой пропагаторы имеют вид

$$
\widetilde{D}(k)=\frac{g^{\mu \nu}-k^{\mu} k^{\nu} k^{-2}}{k^{2}-m^{2}} .
$$

Некоторые члены $F_{\mu \nu}$, содержащие пропагаторы, не изменятся при таком преобразованиии. Мы напоминаем, что для свободных полей $\partial_{\mu} A_{\mu}^{a}=0$ автоматически. Таким образом, разность между исходным выражением и тем же самым выражением в поперечной калибровке содержит два вида слагаемых:

1) члены, возникающие в результате перехода к поперечной калибровке, их вклад можно представить в виде

$$
\begin{aligned}
& \epsilon^{a b c} \partial_{\mu} A_{\nu}^{b}(x) \partial_{\nu} A_{\mu}^{c}(x) \epsilon^{a d e} \partial_{\rho} A_{\sigma}^{d}(y) \partial_{\sigma} A_{\rho}^{e}(y) D_{c}^{0}(x-y)= \\
& \quad=\left(\partial_{\mu} A_{\nu}^{b}(x) \partial_{\rho} A_{\sigma}^{b}(y)\right)\left(\partial_{\nu} A_{\mu}^{c}(x) \partial_{\sigma} A_{\rho}^{c}(y)\right)-(\sigma \leftrightarrow \rho, \rho \leftrightarrow \sigma)=0 ;
\end{aligned}
$$


2 дополнительные члены в лагранжиане (6), которые порождают слагаемое $\widetilde{S}_{1}^{*}(x) \widetilde{S}_{1}(y)+\widetilde{S}_{1}^{*}(y) \widetilde{S}_{1}(x)$, в точности компенсирующее член $\widetilde{S}_{2}^{*}+\widetilde{S}_{2}$, в результате условие унитарности принимает стандартный вид; здесь $\widetilde{S}$ описывает процесс, обусловленный “дополнительными” членами в лагранжиане (6):

$$
\widetilde{S}_{2}^{*}(x, y)+\widetilde{S}_{2}(x, y)+\widetilde{S}_{1}^{*}(x) \widetilde{S}_{1}(y)+\widetilde{S}_{1}^{*}(y) \widetilde{S}_{1}(x)=0
$$

Таким образом, стандартное условие унитарности выполнено. Именно этот факт мы хотели доказать. В действительности мы продемонстрировали уничтожение полюса при нулевой массе.

Теперь мы покажем, что поля, отвечающие множителю Лагранжа $\lambda$, и духи Фаддеева-Попова также отщепляются. Необходимо иметь в виду, однако, что приведенное здесь доказательство относится только к асимптотическим состояниям. Мы доказали, что поля $\varphi_{ \pm}, b, e$ входят только в векторы с нулевой нормой и, следовательно, не дают вклада в наблюдаемые матричные элементы. Однако, чтобы обеспечить явную перенормируемость, нам могут потребоваться некоторые духовые поля. Например, не нарушая перенормируемости, мы не можем опустить поля $\lambda$ в промежуточных состояниях, так как только их специальная комбинация не нарушает перенормируемости теории.

Эффективное действие (10) инвариантно относительно преобразований, порождаемых генератором $Q_{\text {в }}$ преобразований БРСТ. Это позволяет доказать отщепление полей $\lambda, \bar{c}, c$ от физических полей.

В лагранжиане, описывающем поля $\lambda, \varphi_{ \pm}, b, e, A_{\mu}, c, \bar{c}$, мы сделаем замену переменных

$$
A_{\mu}^{a} \rightarrow \tilde{A}_{\mu}^{a}-\frac{1}{m} \partial_{\mu} \lambda^{a}
$$

которая уничтожает перекрестный член для полей $\lambda^{a}, A_{\mu}^{a}$, тогда эффективный лагранжиан примет вид

$$
\begin{aligned}
L_{\mathrm{eff}}=- & \frac{1}{4} \widetilde{F}_{\mu \nu}^{a} \widetilde{F}_{\mu \nu}^{a}+\frac{m^{2}}{2}\left(\tilde{A}_{\mu}^{a}\right)^{2}+\left(\partial_{\mu} \lambda^{a}\right)^{2}+\partial_{\mu} \varphi_{+}^{*} \partial_{\mu} \varphi_{-}+ \\
& +\left\{\frac{m}{g}\left(D_{\mu} \tilde{\alpha}_{+}\right)^{*}\left(\partial_{\mu} \tilde{\varphi}_{-}\right)+\frac{m}{g}\left(D_{\mu} \tilde{\alpha}_{-}\right)^{*}\left(\partial_{\mu} \tilde{\varphi}_{+}\right)+\partial_{\mu} b^{*} \partial_{\mu} e+\text { э.c. }\right\}+ \\
& +L_{\operatorname{int}}\left(\tilde{A}_{\mu}^{a}-\frac{1}{m} \partial_{\mu} \lambda^{a}\right)
\end{aligned}
$$

где $\widetilde{F}_{\mu \nu}$ обозначает свободную часть тензора кривизны. В этом выражении мы выписали явно асимптотически инвариантный лагранжиан и обозначили остальные поля как $L_{\text {int }}$. Поскольку поле $\lambda$ не меняется при преобразованиях БРСТ, мы можем описывать асимптотический лагранжиан формулой (10).

Заметим, что поле $\lambda$ входит в (10) с множителем $1 / 2$. Пользуясь уравнениями для полей $\lambda, c, \tilde{A}_{\mu}, \varphi_{ \pm}, b, e$, легко показать что $Q_{\mathrm{B}}^{\text {as }}$ сохраняется и, следовательно, коммутирует с $H^{\text {as }}$,

$$
\left[Q_{\mathrm{B}}^{\mathrm{as}}, H^{\mathrm{as}}\right]=0
$$

Как мы объясняли раньше, это условие приводит к равенствам

$$
\lim _{|t| \rightarrow \infty}\left[Q_{\mathrm{B}}^{\mathrm{as}}, H^{\mathrm{as}}\right]=\left[Q_{\mathrm{B}}, H\right]_{-}, \quad \lim _{|t| \rightarrow \infty}\left[K^{\mathrm{B}}, H^{\mathrm{as}}\right]=\left[K_{\mathrm{B}}, H^{\mathrm{as}}\right]_{-},
$$


где

$$
\lim _{|t| \rightarrow \infty} Q_{\mathrm{B}}=U^{-1} Q_{\mathrm{B}}^{\mathrm{as}} U, \quad \lim _{|t| \rightarrow \infty} K_{\mathrm{B}}=U^{-1} K^{\mathrm{B}} U,
$$

что гарантирует унитарность матрицы рассеяния в подпространстве состояний, аннигилируемых зарядом $Q_{\mathrm{B}}^{\mathrm{as}}$.

Согласно приведенным выше рассуждениям оператор $Q_{\mathrm{B}}^{\text {as }}$ можно записать в виде

$$
Q_{\mathrm{B}}^{\mathrm{as}}=\int d^{3} k\left[a_{\lambda^{a}}(\mathbf{k})^{*} a_{c^{a}}(\mathbf{k})-a_{\lambda^{a}}(\mathbf{k}) a_{c^{a}}^{*}(\mathbf{k})\right] .
$$

Здесь обозначения соответствуют обозначениям для оператора $Q_{\mathrm{S}}^{\mathrm{as}}$. Поскольку операторы $a_{c^{a}}$ и $a_{c^{a}}^{*}$ антикоммутируют, оператор $Q_{\mathrm{B}}^{\mathrm{as}}$ также является нильпотентным. Из унитарности матрицы рассеяния в пространстве векторов, аннигилируемых операторами $Q_{\mathrm{S}}^{\mathrm{as}}, Q_{\mathrm{B}}^{\mathrm{as}}$ и $K^{\mathrm{S}}, K^{\mathrm{B}}$, следует, что любой вектор, аннигилируемый операторами $Q_{\mathrm{S}}^{\mathrm{as}}, Q_{\mathrm{B}}^{\mathrm{as}}$ и одновременно операторами $K^{\mathrm{s}}, K^{\mathrm{B}}$, имеет вид

$$
|\psi\rangle_{\mathrm{ph}}=|\psi\rangle_{\mathrm{YM}}+|N\rangle \text {, }
$$

где $|\psi\rangle_{\text {Үм }}$ это физический вектор в стандартной формулировке массивной неабелевой калибровочной теории, а вектор $|N\rangle$ - это вектор с нулевой нормой. Чтобы доказать полную эквивалентность нашей теории стандартной, нужно факторизовать пространство векторов, аннигилируемых операторами $Q_{\mathrm{B}}, Q_{\mathrm{S}}$, по векторам с нулевой нормой.

\section{4. ЗАКЛЮЧЕНИЕ}

В представленной работе была описана модель, которая не требует для перенормируемости введения наблюдаемых скалярных полей. Тем не менее такие поля могут быть введены. Можно спросить, как это согласуется с работой [17], в которой утверждается, что существует только одна перенормируемая модель неабелевых калибровочных полей, а именно модель Брута-Англера-Хиггса. Здесь нет никакого противоречия, наша модель описывает другую теорию. В частности, в ней не существует калибровки, которая рассматривается в работе [17], а лагранжиан (6) отличается от стандартного лагранжиана Янга-Миллса.

Благодарности. Я хотел бы выразить мою глубокую благодарность Д. Быкову и Л. Фаддееву. Моя особая благодарность А. Квадри за критицизм, который сильно помог мне.

\section{Список литературы}

[1] F. Englert, R. Brout, "Broken symmetry and the mass of gauge vector mesons", Phys. Lett., 13:9 (1964), 321-323.

[2] P. W. Higgs, "Broken symmetries, massless particles and gauge fields", Phys. Lett., 12:2 (1964), 132-133.

[3] S. Weinberg, "A Model of leptons", Phys. Rev. Lett., 19:21 (1967), 1264-1265.

[4] A. Salam, "Weak and electromagnetic interactions", Elementary Particle Physics: Relativistic Groups and Analyticity, Proceedings of the Eighth Nobel Symposium (Aspenäsgarden, Lerum, Sweden, July 19-25, 1968 ed N. Svartholm), Almqvist and Wiksell, Stockholm, 1968, 367-377. 
[5] S.L. Glashow, "Partial-symmetries of weak interactions", Nucl. Phys., 22:4 (1961), $579-588$.

[6] A. A. Slavnov, "A Lorentz invariant formulation of the Yang-Mills theory with gauge invariant ghost field Lagrangian", JHEP, 08 (2008), 047, 11 pp., arXiv: 0807.1795.

[7] А.А. Славнов, "Лоренц-инвариантное квантование теории Янга-Миллса без неоднозначности Грибова", ТМФ, 161:2 (2009), 204-211.

[8] А.А. Славнов, “Лоренц-инвариантное квантование теории Янга-Миллса без неоднозначности Грибова", Тр. МИАН, 272 (2011), 246-255.

[9] A. Quadri, A. A. Slavnov, "Renormalization of the Yang-Mills theory in the ambiguity-free gauge", JHEP, 07 (2010), 087, 22 pp., arXiv: 1002.2490.

[10] А. Квадри, А.А. Славнов, "Свободная от неоднозначности формулировка модели Хиггса-Киббла", ТМФ, 166:3 (2011), 336-349.

[11] А. А. Славнов, “Новый подход к квантованию поля Янга-Миллса", ТМФ, 183:2 (2015), 163-176.

[12] А.А. Славнов, “Квантование моделей массивных неабелевых калибровочных полей со спонтанно нарушенной симметрией вне рамок теории возмущений”, ТМФ, 189:2 (2016), 279-285.

[13] V. N. Gribov, "Quantization of non-Abelian gauge theories", Nucl. Phys. B, 139:1-2 (1978), $1-19$.

[14] К. Ициксон, Ж.-Б. Зюбер, Квантовая теория поля, т. 2, Мир, М., 1984.

[15] Л. Д. Фаддеев, "О разделении эффектов самодействия и рассеяния по теории возмущений”, Докл. АН СССР, 152:3 (1963), 573-576.

[16] А.А. Славнов, Л. Д. Фаддеев, Введение в квантовую теорию калибровочных полей, Наука, М., 1988.

[17] J. M. Cornwall, D. N. Levin, G. Tiktopoulos, "Derivation of gauge invariance from high-energy unitarity bounds on the $S$ matrix", Phys. Rev. D, 10:4 (1963), 1145-1167. 